This article has been published in:

Analyzing Digital Discourse. Patricia Bou-Franch and Pilar Garcés-Conejos Blitvich (eds.). Cham: Palgrave Macmillan, 2019, 219-250.

http://doi.org/10.1007/978-3-319-92663-6_8

If you want to quote from this document, please consult the page numbers in the right hand margins.

8

\title{
Linguistic Expert Creation in Online
} Health Practices

\author{
Marie-Thérèse Rudolf von Rohr, Franziska Thurnherr, \\ and Miriam A. Locher
}

\subsection{Introduction}

In this chapter, we focus on the linguistic creation of expert identities in online health practices. Previous research by, for example, Armstrong, Koteyko, and Powell (2011), Kouper (2010), Gross (2015), Harrison and Barlow (2009), Locher (2006), Rudolf von Rohr (2015), Sillence (2010), Thurnherr, Rudolf von Rohr, and Locher (2016), and Veen, te Molder, Gremmen, and van Woerkum (2010) identified a range of discourse strategies that seem to be commonly used to establish expertise in online health practices, such as referring to other informational sources, referring to one's professional status, listing numerical facts, displaying empathy, using humor, and mobilizing personal narratives. The four data sets examined in the present chapter are an online advice column of an American higher educational institution, email counseling exchanges from a British university, peer forums to quit smoking, and professional

websites for smoking cessation in the UK. In order for their users to take up information or advice, accept support, or remain on site, participants need to position themselves as trustworthy and credible experts in professional as well as peer-to-peer interaction (Harvey \& Koteyko, 2013; Locher, 2013; Richardson, 2003; Sillence \& Briggs, 2015). In the case of the advice column, the advice-giver is a fictional persona named Lucy. The team behind the advisor persona has to use language in such a way that the advice will be taken seriously and will ideally be followed. Regarding email counseling, the trustworthiness of 
the service is a requirement for clients. Further, expertise continues to be negotiated in interaction, as counselors want clients to engage with the support provided. In the case of peer forums to quit smoking, contributors also face several 'rhetorical challenges' that are connected to issues of trust (Harvey \& Koteyko, 2013, p. 165). In the role of helpgivers, contributors have to establish their expertise and credibility to position themselves as trustworthy advice-givers (Eichhorn, 2008; Harvey \& Koteyko, 2013; Richardson, 2003; Rudolf von Rohr, 2015; Thurnherr et al., 2016, etc.). Additionally, clients of email counseling and help-seekers on forums can also position themselves as experts in interaction. Professional websites communicating on smoking cessation face the challenge of being perceived as a trustworthy source, giving expert advice. In other words, studying how expert identities are created in our different e-health contexts is of interest for this chapter. Expertise is especially relevant since it facilitates important activities such as advising and informing in online health practices. We approach expertise from a constructionist point of view. Thus, we see expertise as being multifaceted and discursively constructed. Further, we argue that expertise does not only consist of scientific or experiential knowledge. ${ }^{1}$ Similarly, specific roles in interaction do not necessarily equal access or rights to only one type of expertise (such as laypersons having access to only experiential knowledge). Rather, expertise can refer to scientific, experiential, or personal knowledge, or knowledge of how to interact with a participant to help them manage their health. Moreover, in peer settings, interactants can even validate the knowledge of the entire community when they praise previous help received on the forums and recommend further interaction. Thereby, they simultaneously position the entire group as having expertise and

strengthen their ties to the group. In other words, a gamut of strategies can be utilized in interaction to display various notions of expertise. Our aim is not only to describe the strategies used in the specific practices researched and to compare them to previously reported results, but also to explore their link to contextual factors (social and technological) and how the strategies interplay with each other. In the next section, we will motivate our choice to study the creation of expertise further by reviewing some of the literature.

\subsection{Theoretical background to the study of expertise and identity construction in health, online contexts}

Expertise in medical contexts has been researched in previous studies and yielded interesting results with regard to a wide range of aspects such as power asymmetry, identity construction, and other contextual factors. Health practitioners need to project the identity of credible experts if they want patients to align with the advice and information provided (see Segal, 2005, who argues that expertise equals ethos in health contexts). Being perceived as a credible expert is connected to establishing trust. Sillence, Briggs, Harris, and Fishwick (2006, p. 697) have even suggested that 'trust is key' in online health advice (see also Sillence \& Briggs, 2015). In face-to-face doctor-patient interaction, 
the power asymmetry between patients and medical experts used to be clearly established due to the institutional nature of the interaction (Bigi, 2011). The internet has contributed to a change in the positions available to patients in medical consultations. It has been argued that the information provided on the internet facilitates more symmetrical decision-making between healthcare providers and patients/clients. In this context, the role of accessibility to information has been emphasized as it empowers patients to make responsible health decisions (Heaton, 2011). Lay consumers surfing the Web can potentially access the same scientific knowledge and information as experts on the internet (Sarangi \& Clarke, 2002). Ziebland and Wyke (2012) even argue that '[o]nline resources are now established as a

primary route to health information and support' (p. 220). Thus, lay consumers can independently become more knowledgeable without the guidance of health professionals. Moreover, lay consumers can produce health information themselves (Seale, 2003). In fact, lay expertise in the form of personal experience has gained in authority over the last decade and can be mobilized to counter evidence-based information. Experiential advice and lay expertise seem to be highly valued by consumers surfing the Web (Furedi, 2008; Seale, 2002; Sillence, 2010). If the expertise of health practitioners is potentially in contest with lay sources online, this raises the question of how practitioners construct their expert identities in order to be perceived as trustworthy advice-givers. Moreover, the question of how lay expertise is constructed online also arises; especially considering that laypeople usually cannot rely on institutional backing. If laypeople want to be taken seriously as trustworthy advisors, they need to establish expertise. All of the authors mentioned point toward a vital need to understand how expertise is constructed in an online health context, in which the participants' main goal is to improve someone's well-being.

Three studies are singled out in this review as they are instrumental in illustrating the theoretical background on health communication for the present chapter (Locher, 2006; Richardson, 2003; Sillence \& Briggs, 2015). Sillence and Briggs (2015) explored how people evaluate the trustworthiness of e-health sites from a psychological perspective. They list four factors that shape how internet users develop trust, suggesting these factors be seen as stages of how trust evolves: (1) visual appeal, (2) branding of the site, (3) evaluation of site content, and (4) 'the importance of personalization' and 'relationship management' (Sillence \& Briggs, 2015, p. 472).The first factor refers to the initial decision of whether internet users interact with websites at all, whereas the second is concerned with whether there are trust markers, such as the logos on, or branding of, the site, with which internet users are familiar. These first two factors do not lend themselves for linguistic analysis in the strict sense. Nevertheless, they are essential if we want to consider a holistic picture of how expertise is built up. Also, taking these two factors into account allows us to address the influence of medium and social factors on the practices (Herring, 2007) in our analyses. In a third stage, internet users assess whether they find the information provided to be of good 
quality, believable, and altruistic. Finally, internet users are more likely to develop 'longerterm trust' if they engage in continued interaction where they can self-disclose or if content is personalized, for instance, through 'patient-authored material' (Sillence \& Briggs, 2015, p. 481).

Several studies on written online health discourse have analyzed warranting strategies, that is, discourse strategies used to create expertise and to display credibility ${ }^{2}$ (Richardson, 2003), in order to generate trustworthiness in different practices (e.g. Eichhorn, 2008, on an eating disorder forum; Morrow, 2006, on a forum dealing with depression; and Kouper, 2010, on a LiveJournal community blog about motherhood). Richardson's study of an online newsgroup discussing the risk of cell phones has set the ground for several later studies on the discursive negotiation of expertise and credibility, including ours. In her study of discussions about the health risks of mobile phones in internet newsgroups, she found that participants positioned themselves as credible expert contributors by (1) referring to other sources, (2) referring to their own experience, (3) referring to the expertise of friends, (4) self-categorizing as experts, or (5) using a technical register. Further studies corroborated the strategies Richardson found to enhance credibility and trustworthiness in online health support groups dealing with a range of conditions but also added different ones. For example, presenting key health statistics (a case of using technical register) or requesting further information (a new strategy), not unlike in traditional medical discourse, have been reported in Fage-Butler and Jensen (2013) on informational and relational aspects in a thyroid forum, or in Sillence (2010) on advice in a prostate cancer forum. In particular, the role of how participants share their own experience to position themselves as authoritative sources while simultaneously showing empathy when giving advice has been found an important characteristic of peer-to-peer online health groups (e.g. Fage-Butler \& Jensen, 2013; Kouper, 2010; Sillence \& Briggs, 2015; Wright, 2015; Veen et al., 2010, etc.).

Locher's (2006) work on an online advice column discussed how the expert identity of the advisor Lucy emerged in the online practice in question. In this professional-lay context, Locher (2006) found that seven factors contributed to making up the expert persona Lucy. First, the advisor was mostly referred to by her first name, which boosted her identity as expert but also reminded readers of her fictionality. Second,

Lucy directed readers to other sources, invoked her expert status as health educator, and

appeared to 'quote facts in numbers and percentages' (Locher, 2013, p. 348). She expanded on the original question of the advice-seeker and gave 'detailed background information' (ibid.). Further, Locher (2006, pp. 193-194) suggests that Lucy 'makes readers think and gives options', pointing to the preference for non-directiveness typical in counseling contexts. These strategies can be straightforwardly linked to boosting Lucy's expert status. Moreover, Lucy's response letters were characterized by a register which avoided medical jargon in order to cater to the target audience consisting of students. Further, the advisor Lucy clearly marked her stance on the questioner's actions, which helped her to sanction behavior and attitudes in line with good health information. Additionally, Lucy engaged in relational work, such as the display of empathy as well as 
bonding and hedging through humor. Thereby, she emerged as caring advice-giver who was 'clever and witty' (Locher, 2006, p. 201). Finally, Locher (2013) also points to the importance of the overall visual design, explaining that it clearly marks the advice column as an expert professional source.

These and other studies have informed our theoretical background with regards to the creation of expertise in health discourses. Further, discursive identity construction lends itself especially well to studying how expertise is negotiated in discourse. Thereby, we adopt a constructionist approach to identity and follow Bucholtz and Hall (2005), who argue that identity should be seen as a social, discursive, emergent, and relational phenomenon. In other words, it cannot be captured simply by taking account of variables such as age, gender, education, and so on. We make use of the concept of positioning as developed in discursive psychology (Bucholtz \& Hall, 2005; Davies \& Harré, 1990; Hall \& Bucholtz, 2013). This means that we are interested in how interactants position themselves vis-à-vis each other and how they take each other's face concerns into account during interaction.

Our brief review of the previous literature has, on the one hand, highlighted that there is already an extensive body of research on the discursive creation of expertise and credibility and its link to trust in online health discourse. On the other hand, it also positioned our study within a constructivist approach to the creation of expertise and identity. In view of

the above finding that the creation of expertise is key in e-health contexts, this chapter will try to answer the following research questions for our specific corpus: (1) How do writers create expert identities in health contexts where credibility is important? (2) To what extent does the computer-mediated context shape/facilitate/empower the practices observed?

\subsection{Methodology and data}

Our study aims to add to existing research on expertise by adopting a comprehensive and holistic understanding of its creation and potential resulting trustworthiness from a discourse-analytic perspective. We do so by combining an analysis of the warranting strategies that are used to create expertise, as reported by Richardson (2003) and Locher (2006), with Sillence and Briggs' (2015) model of how trust is established. The extension of Locher's (2006) initial work on an online advice column allows us to reveal not only the types of discursive strategies used to construct expertise, but also how these strategies interact with each other and how certain characteristics of practices, such as interactivity, can influence the use and interplay of strategies. To answer our first research question of how expert identities are created, we thus focus on what kind of, and how, discourse strategies are used to display and negotiate expertise and trustworthiness. In a qualitative process, we identify linguistic strategies by engaging in close readings of the texts. We draw on our prior knowledge of the individual practices (see Locher, 2010; Rudolf von 
Rohr, 2015, 2017; Thurnherr et al., 2016) to identify particular strategies used to display expertise, which can then be put into relation with the construction of an expert identity. Moreover, our second research question, regarding how the computer-mediated communication (CMC) context shapes the practices observed, leads us to touch upon the influence of the differing social and medium factors (Herring, 2007) of the practices examined. Throughout, we highlight how Sillence and Briggs' model (2015) is reflected in the participants' discursive work.

This chapter belongs to a project entitled 'Language and Health Online', which is both a continuation and an expansion of Locher's (2006) study of the advice health column 'Lucy Answers'. We work with

the same theoretical foundations, delving into the discursive setup of online health advice

practices and interpersonal aspects such as credibility and trustworthiness that are associated with it. One of our project's goals consists in being able to expand the initial study to include a range of asynchronous online health advice settings that use different modes (such as email, forums, and less interactive websites) and vary in topic and in participation structure (Herring, 2007). We are working with four data sets that, despite differences in medium and social factors, all have in common that language is at the forefront to communicate on health and instantiate change in interactants. The data sets are introduced considering whether authoritative health professionals are part of the practices and the level of interactivity. An overview is presented in Table 8.1. We work with subsamples for three of the four sets.

Table 8.1. Overview of our four data sets, ordered according to decreasing presence of health professionals and increasing level of interactivity.

\begin{tabular}{|c|c|c|c|}
\hline Data source & Location/sites & Interactants & Source size \\
\hline $\begin{array}{l}\text { Anti-smoking } \\
\text { websites }\end{array}$ & $\begin{array}{l}\text { UK nonprofit and } \\
\text { commercial websites }\end{array}$ & $\begin{array}{l}\text { Professionals, charities, } \\
\text { broad audience }\end{array}$ & $\begin{array}{l}7 \text { websites analyzed } \\
\text { (corresponds to } 23 \% \text { of } \\
\text { larger corpus) }\end{array}$ \\
\hline $\begin{array}{l}\text { Online advice } \\
\text { column }\end{array}$ & $\begin{array}{l}\text { American Uni, health } \\
\text { program }\end{array}$ & $\begin{array}{l}\text { Health team 'Lucy' } \\
\text { Anonymous questioners }\end{array}$ & $\begin{array}{l}280 \text { texts analyzed } \\
\text { (corresponds to } 10 \% \text { of } \\
\text { larger corpus) }\end{array}$ \\
\hline $\begin{array}{l}\text { Email counseling } \\
\text { exchanges }\end{array}$ & $\begin{array}{l}\text { British Uni, counseling } \\
\text { service }\end{array}$ & $\begin{array}{l}1 \text { counselor (BACP } \\
\text { accredited, trained in } \\
\text { online counseling), } 5 \\
\text { clients (students of } \\
\text { British university) }\end{array}$ & $\begin{array}{l}5 \text { threads (this } \\
\text { corresponds to the } \\
\text { entire counseling } \\
\text { corpus) } \\
10-12 \text { emails per thread }\end{array}$ \\
\hline $\begin{array}{l}\text { Online forums } \\
\text { providing peer } \\
\text { support for } \\
\text { smoking quitters }\end{array}$ & $\begin{array}{l}\text { UK non-commerc. peer } \\
\text { support groups: } \\
\text { (a) Smokingisbad; } \\
\text { (b) Nosmokingday.co.uk }\end{array}$ & $\begin{array}{l}\text { Peers, that is, smoking } \\
\text { quitters } \\
\text { Between } 3 \text { and } 14 \\
\text { interactants per thread }\end{array}$ & $\begin{array}{l}27 \text { threads analyzed } \\
\text { (corresponds to } 34 \% \text { of } \\
\text { larger corpus) } \\
\text { Between } 11 \text { and } 19 \\
\text { posts }\end{array}$ \\
\hline
\end{tabular}

The subsample of anti-smoking websites for the present study consists of seven websites (collected in 2012) and is the least interactive of our four sets. ${ }^{3}$ The sources include different institutional and organizational backgrounds (governmental, commercial, and nonprofit) and are all set in the UK. The sample is characterized by its extensive coverage 
of information on smoking cessation, ranging from how smoking affects one's beauty to smoking during pregnancy. Further, the websites of this subsample highlight the importance of smoking cessation services while also offering opportunities for readers to get in contact. Expertise is created in various ways on these multimodal platforms of information (see Herring, 2013), as it is established in different sections, such as Tips sections, FAQs or testimonials. In these subsites, the hierarchy of knowledge between website providers and readers may be more or less emphasized.

The second data set consists of 280 problem and response letters from an online health advice column, which represents 10 percent of the entire corpus (collected in 2002 and 2004). The column is run by professional health experts at an American university who create the fictional agony aunt Lucy. It covers seven topic categories from emotional health to information on drugs and fitness. There is only one exchange between the advice-seeker and the advice-giver. The advice-seekers are entirely anonymous. Previous work on this practice has been published in Locher $(2006,2010)$ and Locher and Hoffmann (2006).

The third set consists of five threads of email between one counselor and five different clients, obtained from a counseling service at a British university in 2013 and 2014. Client and counselor only interact online, that is, they do not meet face-to-face. Each thread consists of 10-12 emails, with themes ranging from relationship issues to depression, anxiety, or stress. The exchanges were carried out in two ways: the counseling content is either in the body of the email (exchanges obtained in 2013) or in a password-protected word document that is sent back and forth between the interactants (exchanges obtained in 2014). In the exchanges, the client and counselor discuss the client's problems, devise coping strategies, and evaluate the client's progress. We have gained retrospective informed consent. In other words, we have asked the interactants for

access to their exchanges only after the counseling was concluded. Finally, the counselor anonymized the exchanges to protect the clients' identity.

The fourth set consists of data from two forums that are open access, noncommercial independent, or charity peer support groups on the topic of smoking cessation, in which participants provide each other with support, advice, and information: (a) SmokingisBad and (b) nosmokingday.co.uk. ${ }^{4}$ The threads from the corpus were posted between March and April 2012 in subforums where participants who had just started a fresh attempt at quitting smoking mainly initiate the interaction. ${ }^{5}$ For this study, we compiled a subcorpus of 27 threads, dealing either with explicit requests for help, support, or advice by initiating participants (15 threads) or with relapse/starting over announcements by initiating participants (12 threads). In requests for help threads, the advice-giving frame, including the asymmetry in knowledge, is brought about by initiators themselves. In contrast, in relapse threads initiating posters are experienced in quitting and often in being a community member and are primarily looking for support. Both contexts raise interesting questions regarding the interpersonal or interactional purpose for which expertise and credibility need to be discursively constructed. In terms of ethics, we did not ask for informed consent, basing our decision on the heuristic principles outlined by the 
Association of Internet Researchers (Ess \& the AolR Ethics Working Committee, 2002; Markham, Buchanan, \& the AolR Ethics Working Committee, 2012). This is because, with respect to the public-private continuum, we observe that interactants place group interaction in the public sphere since they refer to the private messaging function if they want to continue talking about more delicate issues.

In Table 8.2, we compare the individual data sets with respect to three important medium and social factors: interactivity, the number of parties involved, and whether the main participants were health professionals, laypeople, or both. Our notion of interactivity is closely linked to turn-taking, designating the fact that users can interact with each other (see Warnick, 2007, for a discussion of different understandings of interactivity). Data sets were comparable due to their similarity regarding online health concerns and a focus on problem-solving, advising, and information-giving.

Table 8.2. Three important medium and situational factors of the data sample.

\begin{tabular}{|c|c|c|c|}
\hline Data source & Interactivity & Type of interactivity & Participants \\
\hline $\begin{array}{l}\text { Anti-smoking } \\
\text { websites }\end{array}$ & Not interactive & No turn-taking & Professional \\
\hline $\begin{array}{l}\text { Online advice } \\
\text { column }\end{array}$ & Limited interactivity & $\begin{array}{l}\text { Problem letter- } \\
\text { Response letter }\end{array}$ & Professional-lay \\
\hline $\begin{array}{l}\text { Email counseling } \\
\text { exchanges }\end{array}$ & Very interactive & $\begin{array}{l}\text { Two-party interaction } \\
\text { Several turns }\end{array}$ & Professional-lay \\
\hline $\begin{array}{l}\text { Online forums } \\
\text { providing peer } \\
\text { support for } \\
\text { smoking quitters }\end{array}$ & Highly interactive & $\begin{array}{l}\text { Multiparty interaction } \\
\text { Several turns }\end{array}$ & Lay interaction \\
\hline
\end{tabular}

\subsection{Results and discussion}

We established four dimensions of how expertise surfaces in our online practices:

1. Embeddedness of the practice in online health context

2. Interplay between strategies

3. Interactivity of medium

4. Clients/laypeople and professionals equally construct expertise

The first three dimensions are intertwined. This demonstrates how strategies for creating expertise are embedded, act together, and are shaped by the interactivity afforded by the medium. The fourth dimension highlights that it is not just the health professionals who engage in acts of constructing expertise but also laypeople. In what follows, we discuss each of these four dimensions in turn. Due to space constraints, we cannot present examples and detailed analysis for all four data sets in each section. Instead, we will choose one practice per dimension to illustrate our insights and will then summarize how this dimension panned out in the other practices. The sequence of discussion will vary depending on which practice was chosen for illustration. 
It is important to point out that the linguistic strategies we found (to be illustrated in Sect. 8.4.2) are embedded within the framework of the entire online interaction. In our data sets, the visual designs and the use of trust markers are available as pointers of institutional or contextual expertise (Sillence \& Briggs, 2015). To illustrate this, we use the professional anti-smoking websites. The branding and visual design is highly dependent on the institutional background of these sources. Governmental websites can invoke their institutional standing by using their well-known logos. Screenshot shows how the UK national anti-smoking website is professionally made with a layout that facilitates information seeking. Moreover, the logos index the institutional background of the NHS (frames top left and top right). This prominent placement at the top conveys trustworthiness to website visitors, possibly encouraging them to stay on the website (Sillence \& Briggs, 2015). The institutional setting is further reinforced by the possibility to call a helpline (frame top center) or to order a smokefree kit (frame bottom right), which demonstrates the professionalism and scope of the service. In combination with these trust markers, advice and information offered on a range of aspects related to smoking cessation position this practice as an informationally founded and noncommercial source.

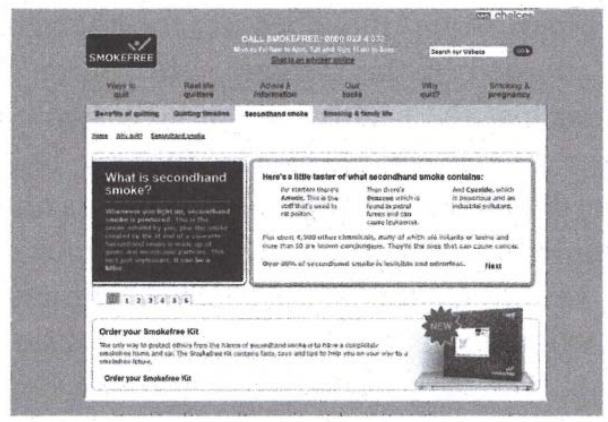

Screenshot: smokefree.nhs.uk, bold framed squares added.

Continuing with professional health sites, we observe that the letter exchange of the advice column Lucy Answers is framed by information about the health service provided by the educational institution to which it belongs. Thereby, it profits from the latter's reputation and standing. Additionally, the hyperlinks to further resources of a medical nature and the logos of the health department and the university give the advice column a professional appearance.

In email counseling, the initial construction of expertise for the counselor precedes the first email exchange. Clients approach the counselor via the university health website. The website is verified as a university service, displayed by the specific URL containing the university's web address, the university's official logo, and the general style of the site with regards to font, colors, and other design features that are in line with the rest of the university's website. Additionally, the overall counseling service is described as accredited, professional, and confidential on the introductory page and each counselor has their 
professional credentials listed separately on a subpage. Finally, the client is asked to fill in a questionnaire regarding their well-being, indicating that clients and their issues are taken seriously and are assessed in a professional manner from the start of the interaction.

In nonprofessional interaction between lay participants in smoking cessation forums, we can also observe embeddedness in a larger context. First, the thematic direction of the forum embeds the interaction in a health context. While there is no institutional backing of individual interactants, there is system generated information about how often a contributor is active in the forum (i.e. the number of posts made per contributor overall are listed next to the date of the post). In addition, participants get titles depending on their number of posts ('senior, junior member', indicated as labels under the user name). This can indirectly be seen as information that gives them expert status. Further, initial posters to early subforums Day1 or Day2 automatically position themselves as being at the beginning of their quitting journey thus presenting themselves as in need of help or as being less experienced in quitting. These various design features and strategies highlight what Sillence and Briggs (2015) termed 'visual appeal' and 'the branding of the site' ( $p$. 472). While the logos referring to the institutional backing of some of our

practices are more evident in the construction of expertise, we also see system-given information in the lay forums that contribute to the creation of expertise. The design features and strategies thus serve to create identities of experts who are credible, and may serve as the first step in establishing a trusting relationship between help-seekers and helpers.

\subsubsection{Expert identity strategies and their interplay}

Secondly, we focus on the linguistic strategies used for the creation of expertise. Table 8.3 lists the recurring strategies that we identified as contributing to identity construction in our various practices. Each of our data sets has a particular combination of strategies that is unique and can also change over time or in location (e.g., on the website and within the thread). The crosses indicate presence of strategies. They are organized into six groups to show that some of these strategies are related to a specific aspect of problem-solving. Expertise is brought to the fore in some groups more clearly than in others, especially the 'information-related' group might seem logically connected to constructing expertise. However, the other strategies also contribute to an expert identity. We decided to classify strategies into groups according to what we judged to be their primary function in the interactions. The table is necessarily a simplification of what occurs in interaction, as some strategies could be attributed to more than one group of strategies. Together the strategies function to establish a particular impression that may - of course - differ in various readers' minds.

We will briefly illustrate some of the strategies employed by the health team to create the agony aunt Lucy. Lucy makes use of all the strategies marked with [x] in Table 8.3, column 3. And we argue that it is in fact the interplay of these strategies that creates a credible and trustworthy expert identity appropriate for this particular context (i.e. an 
online health column aimed at college students). First, it is noteworthy that Lucy refers to herself in the third person (Examples 1 and 2):

(1) Lucy assumes that this is what you mean by contamination. (LA, drugs)

(2) Lucy noticed that you signed your letter 'argh' and wondered about that. (LA, sexuality)

Table 8.3. List of recurring expert identity strategies.

\begin{tabular}{|c|c|c|c|c|c|}
\hline & \multirow{2}{*}{$\begin{array}{l}\text { Smoking } \\
\text { cessation } \\
\text { websites }\end{array}$} & \multirow{2}{*}{$\begin{array}{l}\text { Lucy in } \\
\text { Lucy } \\
\text { Answers }\end{array}$} & \multicolumn{2}{|c|}{ Email counseling $^{a}$} & \multirow{2}{*}{$\begin{array}{l}\text { Smoking } \\
\text { cessation } \\
\text { forums }\end{array}$} \\
\hline & & & Counselor & Client & \\
\hline \multicolumn{6}{|l|}{ Status related: } \\
\hline $\begin{array}{l}\text { Name, self-reference, address terms } \\
\text { Information related: }\end{array}$ & $x$ & $x$ & $x$ & & $x$ \\
\hline $\begin{array}{l}\text { Competent and knowledgeable source of } \\
\text { accurate information }\end{array}$ & $x$ & $\mathrm{x}$ & $x$ & $x$ & $\mathrm{x}$ \\
\hline Referring to further help & $x$ & $x$ & $x$ & & $x$ \\
\hline Presence/absence of jargon & $x$ & $x$ & $x$ & $x$ & \\
\hline Predicting future developments & $x$ & $x$ & $x$ & & $x$ \\
\hline \multicolumn{6}{|l|}{ Advice related: } \\
\hline Providing strategies step-by-step & $x$ & $x$ & $x$ & & $x$ \\
\hline $\begin{array}{l}\text { Making the addressee think and giving } \\
\text { options }\end{array}$ & $x$ & $\mathrm{x}$ & $x$ & & $\mathrm{x}$ \\
\hline \multicolumn{6}{|l|}{ Assessment related: } \\
\hline Having an opinion (praise and criticism) & $x$ & $x$ & $x$ & $x$ & $x$ \\
\hline \multicolumn{6}{|l|}{ Interpersonal: } \\
\hline Showing empathy & $x$ & $x$ & $x$ & & $x$ \\
\hline Displaying a sense of humor & & $x$ & $x$ & & \\
\hline $\begin{array}{l}\text { Showing unconditional positive regard } \\
\text { (UPR) }\end{array}$ & & $x$ & $x$ & & $x$ \\
\hline Showing active listening & & & $x$ & & \\
\hline Being welcoming & & $x$ & $x$ & & $x$ \\
\hline $\begin{array}{l}\text { Expressing confidence in someone's } \\
\text { abilities }\end{array}$ & $x$ & $x$ & $x$ & $x$ & $x$ \\
\hline
\end{tabular}

Table 8.3. (continued)

\begin{tabular}{|c|c|c|c|c|c|}
\hline & \multirow{2}{*}{$\begin{array}{l}\text { Smoking } \\
\text { cessation } \\
\text { websites }\end{array}$} & \multirow{2}{*}{$\begin{array}{l}\text { Lucy in } \\
\text { Lucy } \\
\text { Answers }\end{array}$} & \multicolumn{2}{|c|}{ Email counseling $^{\mathrm{a}}$} & \multirow{2}{*}{$\begin{array}{l}\text { Smoking } \\
\text { cessation } \\
\text { forums }\end{array}$} \\
\hline & & & Counselor & Client & \\
\hline \multicolumn{6}{|l|}{ Interactive: } \\
\hline $\begin{array}{l}\text { Requesting information that only the } \\
\text { advice-seeker has access to }\end{array}$ & & $x$ & $x$ & & $x$ \\
\hline Agreement & $x$ & & $x$ & $x$ & $x$ \\
\hline $\begin{array}{l}\text { Evaluation of a suggested coping } \\
\text { strategy }\end{array}$ & & & $x$ & $x$ & \\
\hline Recommend continued interaction & $x$ & & $x$ & & $x$ \\
\hline
\end{tabular}

\section{Adapted and expanded from Locher (2006)}

${ }^{a}$ We have separated the use of strategies according to interactant in the email corpus, as the counselor and client both construct expertise in different ways and to different ends 
Referring to herself in the third person is a strongly marked feature (see Locher \& Hoffmann, 2006). We argue that this strategy simultaneously serves two functions. First, it is a way to point to the team of health educators that stand behind the pseudonym Lucy. This reinforces her identity as an authoritative expert. At the same time, it is also an honest way of reminding the reader that Lucy is not real. Secondly, the frequent mention of her name writes Lucy into being. This happens only over time, when readers access the site repeatedly.

Lucy's main task is to pass on accurate health information (strategies in group 2) and provide advice (strategies in group 3). The major part of the response texts is thus dedicated to giving information (Example 3), advice (Example 4), and providing further suggestions (Example 4). Consequences of following advice are also often detailed ('predicting future developments').

(3) Panic attacks are periods of heightened anxiety often coupled with an extreme fear of being in crowded or closed places. At first, these attacks are sudden and unexpected, but, if they continue, are often triggered by environment, like going through tunnels, traveling across bridges, or being in crowded elevators. Accompanying symptoms include [etc.] (LA, emotional health)

(4) With counseling for yourself as well, you may be better able to help your partner. If you are at AEI, call Counseling and Psychological Services (CPS) at <phone number>. (LA, emotional health)

While Lucy is clearly positioned as being created by health professionals and belonging to the health services of a university (which legitimizes the practice), she nevertheless avoids medical jargon and chooses an easily accessible, informal, and nonoffensive range of vocabulary items that is suitable for her target audience.

The ways in which advice is rendered (e.g. that Lucy praises and criticizes) and the fact that Lucy invests much into interpersonal strategies such as showing empathy, being welcoming, and displaying humor (not shown in examples) makes her an approachable and human health expert - all this despite the fact that Lucy actually does not exist, but is created by a team of health experts.

Turning to website practices, these use a gamut of strategies for expert identity construction to give advice and information (groups 1-4, as shown in Table 8.3, column 2). While strategies from all groups are employed, Table 8.3 shows that there is fewer use of strategies in the interpersonal and interactive group when compared to the other data sets. Since the websites do not allow users to contribute content, the explanation for the use of fewer strategies is obvious in the interactive group. Sometimes websites compensate for the absence of user interaction in presenting testimonials, which are supposedly personal experiences by real readers. However, we can only speculate as to the reasons for fewer strategies in the interpersonal group. It could be that professional information websites abstain from using interpersonal strategies such as 'show 
unconditional positive regard' or 'display a sense of humor' to avoid misunderstandings and an impression of currying favor.

The strategies used in the email counseling exchanges are manifold, especially on the counselor's side (Table 8.3, column 4). The counselor uses all strategies but one: sharing 'personal experience' is avoided due to rules of professional conduct (Green, 2010). The counselor, therefore, has many different strategies available to construct her expertise, and she uses specific strategies at different times during the exchanges according to changes in her role as advice-giver (see Sect. 8.4.3 for a detailed illustration). Finally, clients (Table 8.3, column 5) use discursive strategies to share knowledge of their own lives on which they are the experts rather than the counselor and position themselves as accurate sources of information (strategy in group 2). Additionally, clients can illustrate their expertise concerning specific coping mechanisms through such strategies as 'having an opinion' (group 4), thereby reporting on progress (for a brief discussion, see Sect. 8.4.4).

A wide range of recurring strategies are employed for expert identity construction in the request and relapsing forum threads, as shown in the list of strategies in Table 8.3, column 6. Mostly, these strategies are employed by responding participants, who, as advice-givers, need to establish their expertise to have their recommendations accepted (strategies from groups 1 to 4). Some strategies such as 'personal experience' and 'express confidence in one's abilities' are also used by initiating participants when they report back on their progress in ongoing interaction. Two strategies, 'be welcoming' and 'recommend continued interaction', do not only contribute to the construction of an individual as expert but reinforce the knowledge and resources of the entire community. The listed strategies need not occur in every individual thread but characterize the practices as a whole.

We cannot illustrate all strategies in detail in this section due to space (see Sects. 8.4.3 and 8.4.4 for an illustration and explanation of several strategies). While our overview of strategies demonstrates that (1) many of those found in our corpora overlap with strategies reported in previous literature, (2) we want to highlight that specific interplays of strategies are utilized to form particular identities in the interactions and are responsible for the successful creation of expertise. Not every interactant in our data used all of them, and it is one of our findings that it is the particular combination of strategies that differentiates our participants in subtle ways.

\subsubsection{Interactivity of medium}

As mentioned in our data description (Sect. 8.3), all four practices differ according to their interactivity. Forums and email counseling are highly interactive and threads extend over several exchanges. Lucy Answers is specifically designed to contain one problem letter and an advisory response,

hence is limited to two turns. Finally, the websites are not interactive in the sense that visitors cannot contribute content. Due to these different degrees of interactivity, we can also witness various ways of constructing expert identities in the four practices. On the 
one hand, there are strategies that are more prone to occur in interactive practices (e.g., 'requesting information that only the advice-seeker has access to', 'agreement', 'evaluation of a suggested coping strategy', and 'recommend continued interaction'). On the other, the continuous interactivity allows for the combination of strategies to change over time in a practice. In other words, while some strategies are favored at the beginning of the interaction, others might be more suitable to create expert identities toward the end of the interaction. In what follows, we will show examples from the email counseling corpus to illustrate interactivity in more detail, before looking into how interactivity influences the construction of expertise in the other three data sets. We will use the concept of (self and other) positioning, derived from discursive psychology (Davies \& Harré, 1990), to point out the stances that interactants take up toward each other.

As a highly interactive medium, email allows client and counselor to exchange multiple messages. The 'interactive' strategies mentioned above are closely connected to the interactivity the medium affords participants. All four practices show use of these strategies, but highly interactive ones utilize them particularly often and with the possibility of further interaction taking place. While the counselor makes use of all four 'interactive' strategies, we will illustrate two with Example (5): 'request information that only the client has access to' and 'agreement'. The client has introduced anxiety as a problematic topic in the first email by stating that she feels anxious and suffers from panic attacks. The counselor confirms that what the client describes are in fact panic attacks and therefore takes up the issue of anxiety:

(5) That certainly sounds like panic attacks Anna, and I'm aware of how frightening they can be, especially when you are away from home and comfort and support of your family, boyfriend, and friends. Help me to understand whet [sic] you have done in these situations to help get through the panic? ${ }^{6}$ Maybe there are some things you have done in the past to cope with anxiety (...). (Counselor: email 2)

By agreeing with the client's self-diagnosis of panic attacks, the counselor positions herself as a knowledgeable source of information about psychological concepts and reclaims the task of diagnosing a mental issue - a task clearly assigned to the expert in the therapeutic relationship. After this confirmation, the counselor shows empathy toward the client by acknowledging the terrifying nature of panic attacks, further highlighting her understanding of the subjective perception of a psychological problem and the client's emotional reaction. Further, the counselor proceeds to request further information of how the client has so far dealt with these panic attacks. The counselor aims to establish what kind of coping mechanisms the client has already in place. These activities diagnosing and assessing a problem, showing understanding of psychological concepts, and asking questions that increase understanding - are tasks closely linked to an expert role. The counselor carries out several such acts of positioning to create her expert advicegiver identity. Interactivity therefore allows the counselor to execute such specific tasks 
and simultaneously create her expertise with the help of strategies that are closely linked to the interactive nature of the medium.

While the counselor might initially use strategies that are closely related to establish her role as advice- and information-giver, we find that the counselor focuses on (aspects of) strategies that position her as an encourager of progress in later stages of the interaction. On the one hand, information-related strategies can, for example, change with regards to content depending on the client's progress. In other words, if the client has improved, the counselor might provide the client with background information on why the application of coping strategies was successful rather than information about the underlying psychological concept that caused the client's problem. On the other, recognizing and assessing progress are foregrounded when the client improves and instructing the client about psychological concepts becomes less important. Example (6) shows an instance in which the counselor praises the client's progress by giving an evaluation of the client's handling of her anxiety:

(6) It sounds like you are making positive progress, and challenging your anxiety, even though it's really tough. (Counselor: email 8 )

The counselor does not need to highlight her own status as a professional counselor anymore, but rather highlights the client's progress. As progress demonstrates the successful work the counselor and client have carried out, the counselor's praise can also be seen as a positive assessment of the suggested coping mechanisms. Recognizing progress and praising become essential tasks of the expert to help clients find and continue to use coping techniques. The client reinforces the counselor's accurate interpretation of her progress by responding you're right, I am making progress in email 9. This development finally leads the counselor to further praise Anna and to encourage her to continue on the same track:

(7) This is great progress Anna, and I can hear how you have found the courage to challenge your anxiety, and this has clearly been part of what has helped you move forward. (...) Keep on doing what you doing, and you will continue to progress :-) (Counselor: email 12)

After the client has established herself as successfully applying suggested coping techniques, the counselor confirms the client's improvement by praising the client's progress. Hence, the counselor positions herself as an encourager. As she was responsible for suggesting the specific coping techniques, she simultaneously reaffirms her expertise as successful advice-giver. Further, she expresses her confidence in the client's abilities to continue to progress. Thus, the counselor has moved from using a combination of strategies that focused more on establishing her position as advice- and information-giver to a combination that centers on interactive and interpersonal aspects to foreground the clients' progress. This move is not random but rather reflects the clients' needs of specific support at different stages of the counseling process. 
In sum, the interactivity of the medium can influence the construction of expertise in two ways: first, due to the fact that interactants can respond in following turns, the use of specific strategies that rely on continued interaction, such as to 'request information only the advice-seeker has access to', is facilitated. Second, the possibility of several exchanges enables continuous interactions over space and time, in which the use or combination of expert strategies can change. Both aspects can be subsumed under Sillence and Briggs' (2015) fourth point concerning the evolvement of trust: 'relationship management' ( $p$. 472).

Turning to the other practices in sequence of increasing interactivity: we find smoking cessation websites offer no options for users to directly interact with other users. Nonetheless, website providers encourage their users to get in contact with them via other communicative means (email, chat), that is, they 'recommend continued interaction' (see Table 8.3). Thereby, they try to move communication into a one-to-one setting. Moreover, while the interplay of expertise strategies changes over time in highly interactive mediums (see email counseling and forums), website providers use different patterns of strategies depending on the section of the websites. Our analysis showed that subsites such as FAQs make extensive use of information- and status-related strategies but relatively little of interpersonal ones. In contrast, sections that are explicitly related to advice-giving (e.g. Stop Smoking tips) prefer to employ advice-related and interpersonal strategies.

In the case of the less interactive practice Lucy Answers, interactivity is limited to a problem and response letter format in Lucy Answers. Strikingly, Lucy still uses one of the interactive strategies to create expertise, namely to request further information from the advice-seeker. While the advice-seeker is not able to respond due to the format of the interaction, Lucy still asks questions aimed at making the reader think. In more interactive mediums, such as the counseling data, these questions could readily be answered, and might also be expected to be answered by clients. In Lucy Answers, the questions are used rhetorically and remind the readers of a dialogue format despite the system's limited interactivity.

Finally, forums are highly interactive, and posters use interactive strategies to construct their own or each other's expertise. For instance, they employ 'agreement' to align with the posters to either boost their own claim or to bond with them, creating group expertise. Respondents can also 'request more information' to properly assess initiators' situation, thus adopting an identity of conscientious and considerate advice-givers. Moreover, the topic of threads seems to influence the extent to which interactive strategies are used. In passages requesting help, interactive strategies are more prominent, suggesting that giving tailored and targeted advice is part of constructing the identity of an expert advicegiver. In contrast, in relapsing, there is a clear preference for interpersonal over interactive strategies and for assessment- to advice-related

ones. This highlights the increased relational needs of initiators in the difficult situation, 241 which advice-givers acknowledge and for which they show their understanding. 
Not only professionals but also clients in counseling and lay interactants in forums construct themselves as experts and authorities both when asking for and when giving advice. We will illustrate this by drawing on the forum data. In the absence of institutional backing, advice-givers (respondents) index their individual expertise with the 15 strategies mentioned in Table 8.3, column 6. They thus write their expertise into being with the aim that advice-seekers consider them trustworthy and comply with their recommendations. Respondents also signal their expertise as community members when they reply to newbies. Moreover, the interactive nature of forum interactions allows clients and initial posters to gradually build up expertise. Hereafter, we use excerpts from a sample thread from the requesting help corpus to illuminate how lay participants interactively construct their own and each other's identities as experts, which is common in the entire forum corpus.

In the sample thread, an initiator asks whether her experience of quitting is normal (Example 8):

(8) Initiator - first post:

I have just past 3 days and 10 hours since my last smoke but is seems more like 3 months. Is it usual for the time to 'drag' on like this...

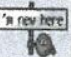

(Requesting help, t20, post 1 )

By sharing her stopping smoking statistics (past 3 days...), implying that quitting is a struggle (more like 3 months), and positioning herself as a new member (smiley), she takes on the identity of a credible participant in the sense that she has a legitimate right to contribute to the forum. Further, she adopts the position of an advice-seeker by her request for normalization (is it usual). At the same time, potential respondents are constructed as experts, since the initiator wants an evaluation based on their experience. Finally, her question opens up an adjacency pair which other members are expected to complete. Her question results in several responses before she posts again. The technical nature of the forums thus allows an immediate reaction by respondent 1 . He establishes similarity between himself and the advice-seeker, a move that works to enhance his credibility and, possibly, his chance to influence the initiator (see also Wright, 2015, on how similarity between interactants is beneficial to the persuasiveness of a sender).

(9) Respondent 1:

I'm also on day 3 [name].

Yes it does seem slow but try to busy yourself and dont [sic] get bored whatever you do.

$[\ldots]$

You will have lots of support on here if you can log on regularly but stay strong and positive!

You can do this! (Requesting help, t20, post 2) 
Respondent 1 fills the second part of the adjacency pair by agreeing with the initiator, which normalizes her concern (Yes it does seem slow). Thereby, respondent 1 accepts the identity of expert quitter even though his initial statement hedges his expertise to a certain extent, since he is no further along in his quitting journey. After having pointed to his position of expertise and the similarity between him and the initiator, the respondent can 'give options' on coping as credible advice-giver (try to..., don't, etc.). Moreover, respondent 1 'recommends continued interaction' with the forum. His use of the willfuture indicates that he has already received lots of support, which stresses his experienced quitter identity, highlights group expertise, and reinforces the authority of the community.

The interactive nature of the forum allows respondents to back up other contributors across posts through 'agreement'. Thus, respondents engage in co-constructing each other's expertise as advice-givers in interaction. In Example (10), the respondent refers to previous posts to introduce her normalizing statement (As others have said):

(10) Respondent 2:

Well done on 3 days and a huge welcome to the forum. As others have said its perfectly normal to feel like times crawling. I noticed it quite a lot when I first quit and found it dificult [sic] but stick with it and occupy yourself and you'll soon feel like times flying by nicely. There's loads of info on the forum to help with your quit and post often and let us know how you're doing. We're all here doing the same thing and support you. (Requesting help, t20, post 6)

By agreeing with earlier respondents, respondent 2 simultaneously legitimizes preceding and her own assessments. Further, she uses her 'personal experience' to position herself as an expert quitter who felt like the initiator in terms of time being slow (noticed it quite a lot). The use of the past tense implies that the respondent has in fact overcome this initial stage without having to explicitly say so. This strengthens her ensuing advice and especially attests to her 'prediction of future developments' (you'll soon feel like). Finally, respondent 2 draws the initiator's attention to the forum as an archive of information (loads of info on the forum), which is only possible due to the persistence of transcript (see Herring, 2007). Moreover, it constructs the online group as having a history of helping contributors, institutionalizing its expertise and authority. To conclude her post, the respondent 'recommend[s] continued interaction' with the community (let us know). She adopts a voice for the entire group, which is indexed by the use of the first-person plural, clearly marking her as a representative member. The use of the plural makes the respondent's bonding attempt more powerful and possibly more effective in tying the initiator to the group (We're all here doing the same thing). This type of involvement and proffer of support is inextricably linked to the user-to-user interactivity on forums.

The initiator returns to the conversation after five posts. She thanks contributors, which indicates that she accepts their stance as experts but also positions herself as an authentic advice-seeker who wants to take up advice-givers' suggestions (thank you all so 
much). Further, she reacts to respondents' recommendation of continued interaction with the forum. In fact, she reifies the group and gives it additional authority and expertise by referring to the community as a space (place to be). In the second paragraph, we can observe how the interactivity of forums allows participants to continuously modify their identity. While the initiator positions herself as struggling in her initial post, she returns and constructs herself as persevering and proud of her success so far.

(11) Initiator - second post:

Oh WOW ... thank you all so much [...]! I'm sure this is the place to be to find support when I think I'm going to fall off the wagon!! I quit on Friday at approx $3 \mathrm{pm}$, and it is now Tuesday 11.30 - so am I still on day 3 or have I moved to day 4 yet? I am quite proud of myself (Requesting help, t20, post 7)

To sum up, this sequence of examples is illustrative of how lay participants position themselves and each other as experts and peers in matters of quitting smoking. It is important to maintain that there is no fixed division in status between advice-givers and advice-seekers: these roles are continuously reassigned and renegotiated in each new thread. Moreover, advice-seekers can adopt a more experienced identity within the interaction of the thread itself. Further, in the context of online health support groups, the expertise of the entire community can be mobilized rhetorically in interaction, especially to involve new members. Contributors can tap into the experience and authority of the entire group by indexing their community membership.

We now turn to the other practices in sequence of decreasing interactivity. In the counseling exchanges, clients also point toward their expertise. On the one hand, they are experts on their own lives and experiences and share details on this knowledge with the counselor. The counselor co-constructs this identity when requesting information that only clients have access to. Additionally, clients also construct themselves as experts on applications of specific coping techniques. After the client agrees with the progress recognized by the counselor in Example (6), she illustrates this progress by narrating how she dealt with missing a flight by writing [I] managed to calm myself down relatively quickly by thinking logically. She therefore positions herself as an expert in applying the coping technique she developed with the counselor's support (for further information on narratives and their use for identity construction, see Thurnherr et al., 2016).

In Lucy Answers, problem letter writers rarely construct themselves as experts. However, they use strategies to establish their credibility as advice-seekers, such as sharing their background, describing symptoms, and requesting help. Due to the format of the advice-column, which only allows for limited interactivity, problem letter writers cannot report on their progress regarding their initial problems. Even so, the way Lucy gives nondirective optional advice assigns problem letter writers an identity as active selfhelpers, who know what is best for them. 
In the smoking cessation websites, laypeople are shown as experts in quitting smoking in the case of testimonials. The success stories of ordinary smokers becoming smoke-free are used to serve as inspiration and example to readers who are positioned as being in the process of quitting smoking. The fact that there is a specifically designated space where the transformation of other lay smokers into nonsmokers is illustrated has a rhetorical function (see Thurnherr et al., 2016). On the one hand, it provides a basis for identification for readers, who may consider testimonials as giving them a motivational spur. On the other, testimonials help to construct the expertise of the smoking cessation websites since successful quitters vouch for the services provided.

\subsection{Conclusions}

In this chapter, we explored four different online health practices and asked the following two research questions:

1. How do writers create expert identities in health contexts where credibility is important?

2. To what extent does the computer-mediated context shape/facilitate/empower the practices observed?

To answer question 1, we found that we could not stop at simply listing a set of strategies (as shown in Table 8.3), but need to include both the wider context in which they are employed and the possibility for interactivity and the ensuing dynamics. Our results thus show that four aspects are vital to the use of these discursive strategies: the embeddedness

of the strategies in the online health context (e.g. as part of an NHS-supported smoking

cessation website), the interplay between strategies (i.e. each practice has its particular combination of strategies), the interactivity of the medium (e.g. there are specific patterns of strategy use according to the stage of counseling), and the fact that not only professionals, but also clients and laypeople construct their expertise (such as peers in an online support group by referring to their own experience). Results revealed that previously found strategies to create expertise also occur in our corpus. Additionally, the findings showed that the interplay of several strategies is in fact needed to explain the creation of credible and trustworthy expert identities for all participants involved. This interplay of strategies varies according to the practice in which expertise is made relevant. Moreover, expert identities are always created in relation to interactants, as evident in the forums where interactants position themselves as experts by answering to advice-seekers' posts. Further, interactants also create expertise for the other interactants, such as clients positioning the counselor as having expertise on how to deal with a specific troubling situation. The discursive approach to expertise especially highlights these interpersonal dimensions of creating expertise. Finally, the interplay of strategies can change (a) over 
time in highly interactive practices and (b) depending on where they occur in less interactive practices.

The discourse-analytic approach of close qualitative analyses to four online practices revealed a complex pattern of highly interdependent strategies for creating linguistic expertise for both lay and professional interactants. The fact that we are dealing with online practices became especially salient when taking the different levels of interactivity into account as well as the persistence of the written data.

In choosing health contexts for our analysis, we may have tapped into a field in which the creation and negotiation of expertise can be expected. This is especially the case since we are dealing with advisory situations. In future research, the question of how authority, credibility, and the potentially resulting trust are intertwined bears further scrutiny. In addition, other, nonhealth-related contexts should be explored for comparison.

\section{Acknowledgments}

We wish to thank the Swiss National Science Foundation for funding the project 'Language and Health Online' (100016_14328611) and especially the counselor and clients who consented to be part of this study. We also thank Patricia Bou-Franch and Pilar Garcés-Conejos Blitvich, who gave us the opportunity to present an early version of this work in Valencia at the first ADDA conference, and the reviewers for their constructive feedback.

\section{Notes}

${ }^{1}$ In contrast to Prior (2003), which focuses on doctor-patient interaction and stresses on professional expertise, we want to highlight that expertise is a multifaceted concept.

${ }^{2}$ It is worth while to point out that the notions of expertise and credibility can sometimes become muddled. We follow Sillence (2010), who argues that showing expertise is one way of establishing credibility and trust.

${ }^{3}$ We work with a bigger corpus of 30 sources in the larger project.

${ }^{4}$ To safeguard the confidentiality of participants, we refer to the first online support group using a pseudonym, SmokingisBad. For copyright reasons, we refer to the second group's original address. However, names and location markers have been changed for both groups. In the meantime, both groups have ceased to exist in the form studied.

${ }^{5}$ Nonetheless, more experienced quitters also post in subforums dealing with the early stages of quitting.

${ }^{6}$ Italics in the original: the counselor mentions in the introductory statement in her first response to the client that any text passages that she writes in italics should be understood as questions that she would like the client to answer. To stay as true to the data as possible, we have decided to keep the italics in the examples presented in this chapter. 


\section{References}

Armstrong, N., Koteyko, N., \& Powell, J. (2011). 'Oh dear, should I really be saying that on here?': Issues of identity and authority in an online diabetes community. Health, 16(4), 1-19. https://doi.org/10.1177/1363459311425514

Bigi, S. (2011). The persuasive role of ethos in doctor-patient interactions. Communication \& Medicine, 8(1), 67-76.

Bucholtz, M., \& Hall, K. (2005). Identity and interaction: A sociocultural linguistic approach. Discourse Studies, 7(4-5), 585-614.

Davies, B., \& Harré, R. (1990). Positioning: The discursive production of selves. Journal for the Theory of Social Behaviour, 20(1), 43-63.

Eichhorn, K. C. (2008). Soliciting and providing social support over the Internet: An investigation of online eating disorder support groups. Journal of Computer-Mediated Communication, 14(1), 67-78. https://doi.org/10.1111/j.1083-6101.2008.01431.x

Ess, C., \& the AolR Ethics Working Committee. (2002). Ethical decision-making and Internet research: Recommendations from the AolR Ethics Working Committee. Approved by AolR, November 27, 2002. Retrieved from http://www.aoir.org/reports/ethics.pdf

Fage-Butler, A.-M., \& Jensen, M. N. (2013). The interpersonal dimension of online patient forums: How patients manage informational and relational aspects in response to posted questions. Hermes, 51, 21-38.

Furedi, F. (2008). Medicalisation in therapy culture. In D. Wainwright (Ed.), A sociology of health (pp. 97-114). Los Angeles: Sage.

Green, J. (2010). Creating the therapeutic relationship in counselling and psychotherapy. Exeter: Learning Matters.

Gross, A. (2015). Asymmetrie und (Patienten-)Expertise in der HIV-Sprechstunde. In A. Busch \& T. Spranz-Fogasy (Eds.), Handbuch Sprache in der Medizin (pp. 282-299). Berlin and Boston: de Gruyter.

Hall, K., \& Bucholtz, M. (2013). Epilogue: Facing identity. Journal of Politeness Research, 9(1), 123-132. https://doi.org/10.1515/pr-2013-0006

Harrison, S., \& Barlow, J. (2009). Politeness strategies and advice-giving in an online arthritis workshop. Journal of Politeness Research, 5(1), 93-111.

https://doi.org/10.1515/JPLR.2009.006

Harvey, K., \& Koteyko, N. (2013). Exploring health communication: Language in action. London: Routledge.

Heaton, L. (2011). Internet and health communication. In M. Consalvo \& C. Ess (Eds.), The handbook of internet studies (pp. 212-231). Malden, MA: Wiley-Blackwell.

Herring, S. C. (2007). A faceted classification scheme for computer-mediated discourse. Language@Internet, 4. Retrieved from http://www.languageatinternet.org/articles/2007/761 Herring, S. C. (2013). Discourse in Web 2.0: Familiar, reconfigured, and emergent. In D. Tannen \& A. M. Trester (Eds.), Georgetown University Round Table on languages and linguistics 2011: Discourse 2.0: Language and new media (pp. 1-25). Washington, DC: Georgetown University Press. 
Locher, M. A. (2006). Advice online: Advice-giving in an American Internet health column. Amsterdam: Benjamins.

Locher, M. A. (2010). Health Internet sites: A linguistic perspective on health advice columns. Social Semiotics, 20(1), 43-59. http://www.tandfonline.com/doi/pdf/10.1080/10350330903438402

Locher, M. A. (2013). Internet advice. In S. Herring, D. Stein, \& T. Virtanen (Eds.), Pragmatics of computer-mediated communication (pp. 339-362). Berlin: Mouton de Gruyter.

Locher, M. A., \& Hoffmann, S. (2006). The emergence of the identity of a fictional expert advicegiver in an American Internet advice column. Text and Talk, 26(1), 69-106. https://doi.org/10.1515/TEXT.2006.004

Markham, A., Buchanan, E., \& the AolR Ethics Working Committee. (2012). Ethical decisionmaking and Internet research recommendations from the AolR Ethics Working Committee (Version 2.0). Retrieved from http://www.aoir.org/reports/ethics2.pdf

Morrow, P. R. (2006). Telling about problems and giving advice in an Internet discussion forum: Some discourse features. Discourse Studies, 8(4), 531-548.

NHS SmokeFree. Retrieved May 1, 2012, from http://www.smokefree.nhs.uk

No Smoking Day Stop Smoking Forum. Retrieved May 1, 2012, from http://www.nosmokingday.co.uk

Prior, L. (2003). Belief, knowledge and expertise: The emergence of the lay expert in medical sociology. Sociology of Health and Illness, 25, 41-57.

Richardson, K. P. (2003). Health risks on the internet: Establishing credibility on line. Health, Risk and Society, 5(2), 171-184.

Rudolf von Rohr, M.-T. (2015). "You will be glad you hung onto this quit": Sharing information and giving support when stopping smoking online. In C. A. Smith \& A. Keselman (Eds.), Meeting health information needs outside of healthcare: Opportunities and challenges (pp. 263-290). Waltham, MA: Chandos/Elsevier.

Rudolf von Rohr, M.-T. (2017). Persuasion in smoking cessation online: An interpersonal pragmatic perspective. Unpublished doctoral dissertation, University of Basel, Basel, Switzerland.

Sarangi, S., \& Clarke, A. (2002). Zones of expertise and the management of uncertainty in genetics risk communication. Research on Language and Social Interaction, 35(2), 139-171.

Seale, C. (2002). Media and health. London: Sage Publications.

Seale, C. (2003). Health and media: An overview. Sociology of Health \& Illness, 25(6), 513-531. https://doi.org/10.1111/1467-9566.t01-1-00356

Segal, J. Z. (2005). Health and the rhetoric of medicine. Carbondale: Southern Illinois University Press.

Sillence, E. (2010). Seeking out very like minded others: Exploring trust and advice issues in an online health support group. International Journal of Web Based Communities, 6(4), 376-394.

Sillence, E., \& Briggs, P. (2015). Trust and engagement in online health: A timeline approach. In S. S. Sundar (Ed.), The handbook of the psychology of communication technology (pp. 469-487). Chichester: Wiley Blackwell.

Sillence, E., Briggs, P., Harris, P., \& Fishwick, L. (2006). A framework for understanding trust factors in web-based health advice. International Journal of Human-Computer Studies, 64(8), 697-713. https://doi.org/10.1016/j.ijhcs.2006.02.007 
Thurnherr, F., Rudolf von Rohr, M.-T., \& Locher, M. A. (2016). The functions of narrative passages in three written online health contexts. Open Linguistics, 2(2), 450-470. https://doi.org/10.1515/opli-2016-0024

Veen, M., te Molder, H., Gremmen, B., \& van Woerkum, C. (2010). Quitting is not an option: An analysis of online diet talk between celiac disease patients. Health, 14(1), 23-40. https://doi.org/10.1177/1363459309347478

Warnick, B. (2007). Rhetoric online: Persuasion and politics on the world wide web. New York; Bern etc.: Peter Lang.

Wright, K. B. (2015). Computer-mediated support for health outcomes: Psychological influences on support processes. In S. S. Sundar (Ed.), The handbook of the psychology of communication technology (pp. 488-506). Chichester: Wiley Blackwell.

Ziebland, S., \& Wyke, S. (2012). Health and illness in a connected world: How might sharing experiences on the Internet affect people's health? The Milbank Quarterly, 90(2), 219-249. 